\title{
Aircraft Trajectory Uncertainties Optimization Based On Interval Analysis Method
}

\author{
Yao Zhu', a, Zhiping Qiu1, Xianjia Chen ${ }^{1, b}$ and Xinrong Liü, c \\ ${ }^{1}$ School of Aeronautic Science and Engineering, Beihang University, Beijing 100191, China; \\ ${ }^{2}$ MAIAA Lab, French Civil Aviation University, TOULOUSE 31055, France. \\ ayaozhu537@gmail.com, bchenxianjia@aliyun.com, cxinrong6914@gmail.com
}

Keywords: trajectory optimization, interval analysis, Bellman method, uncertainty, interval quantification.

\begin{abstract}
Aircraft trajectory planning under wind effect is a critical issue to save fuel consumption and to reduce flight time. However, wind as a kind of natural phenomenon has great uncertainties, which increases the difficulty of trajectory optimization. This paper proposes a aircraft trajectory optimization method which is base on interval analysis method under wind uncertainties. This method uses weather forecast information, based on Gray mathematical theory interval quantification to determinate the interval of the wind speed on every wind grid points. By combining interval analysis method and Bellman method, the minimal flight time consumption trajectory interval is found. Finally, this method is successfully applied on North Atlantic Ocean. Compared with tradition probability method, this method dramatically reduces the optimization time and improves the computational efficiency.
\end{abstract}

\section{Introduction}

With the development of air traffic system, the flights only show the compliance with the regulation cannot satisfy the airlines and passenger. In the past 20 years, aviation oil prices had increased dramatically. Modern aviation industry started to pay attention to pursue more efficient flights. For instance, airlines started to focus on reducing fuel and time consumptions during daily flight operations.

To plan the optimal aircraft trajectory, wind is a critical issue. Airlines endeavor to minimize the adverse effects of headwinds and maximize the beneficial effect of tailwind when planning flight trajectories. The jet streams of the world are in the east-west direction instead of the north-south direction. Therefore, the flights of east-west routes are affected more considerably by en-route winds than that of north-south routes. In order to perceive the best flight performance in terms of the flight time and fuel consumption, airlines may adjust the flight trajectories based on en-route wind profiles. However, it is difficult to find stable trajectories in such complex en-route wind profiles. As a kind of natural phenomenon, wind has great uncertainties. For instance, the directions and speeds of wind vary in different regions, at different altitudes and different times.

Optimizing trajectories is a rich and dynamic research domain. According to practical problems, issues need to be taken into account are different. Here, we are focusing on find the global optimal trajectory in presence of currents in a two dimensional space. Several methods, such as Dijkstra algorithm [1] or $\mathrm{A}^{*}$ algorithm [2], discretize the domain and work on the generated network to find the optimal path. These algorithms are very efficient but the computed solution is restricted to the network. Some others algorithms work on the continuous space. Those algorithms are based on front propagation methods such as Level Set methods, Fast Marching methods and Ordered Upwind methods [3]. Petres [4] adapts the Fast Marching Method to path planning for Autonomous Underwater Vehicles taking into account underwater currents. Alton [5] uses the Ordered Upwind algorithm with the Semi-Lagrangian method to generate optimal trajectories. However, his algorithm cannot be applied to vehicles featuring behaviors more complex than a linear reaction to currents. Daniel [6] takes wind uncertainties into account when planning aircraft trajectory based on combining 
Bellman algorithm and clustering method. The needs to compute certainty path more than thirty times lead to low efficiency of this method. In addition, the unique outcome may not be found finally.

To deal with the uncertainties problem, interval analysis method has its own advantages. Interval math is developed dramatically since 1996 when Moore's treatise [8] was brought out. And now it becomes a new branch in math domain. In recent years, internal analysis method is widely applied in structure engineering domain to solve the bounded uncertainty problems.[10]

This essay proposes an algorithm to plan optimal aircraft trajectories considering uncertainties of wind directions and speeds. Two main parts construct this algorithm. In the first part, according to weather forecast proposed by Producing Ensemble Prediction, the wind directions and speed interval of each point on the wind grid are determined by applying gray mathematics theory in terms of uncertainty quantification. In the second part, the minimal flight time trajectory interval can be generated thanks to the combination of interval analysis method and Bellman algorithm. Finally, this algorithm is applied successfully over the Atlantic Ocean. Comparing with traditional probability method, this method decreases the optimization times and increases the computing efficiency significantly.

\section{Certainty Optimal Trajectory Computation}

\subsection{Wind Grid Computation and Interpolation.}

We consider a 2-degree of freedom point-mass model of a fixed-wing aircraft flying though the North Atlantic Ocean. As an assumption, only cruise part of the flight is considered not only for the simplicity purposes but also because the cruise part is the majority of the flight. Additionally, we assume that the aircraft is flying at constant flight level. In this paper, we do not take temperature issue into account. As a result, we note that based on those assumptions, more complex problems can be simulated by applying this methodology easily.

We compute the optimal trajectory based on the wind predictions with a classical Bellman algorithm. In order to use Bellman algorithm to solve the problem, we need first to build a wind grid, which stores wind data information.

1) Generate the wind grid: We generate a grid of size $N \times M$ nodes on he North Atlantic Ocean. The area from latitude 30 to latitude 70 and from longitude -90 to longitude 10 is taken into account. Each integer latitude and longitude point is regarded as a node. In order to generate smooth trajectories, we divide each latitude and longitude into 20 and 4 boxes. As a result, a $800 \times 400$ grid table is generated.

2) Wind data interpolation: Note that, the wind data only contain the information at integer latitude and longitude node and we need to have the information at all nodes. We use Shepard's Method [9] to do such interpolation.

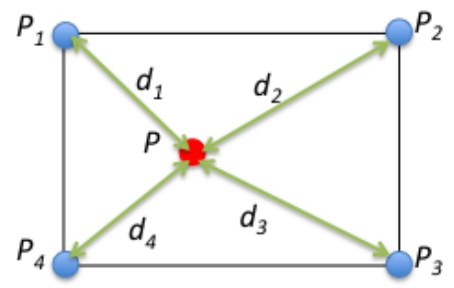

Fig 1. Metric interpolation

Let $F(P)$ be a function of the point $P=(x, y)$ defined for all $P$ in the real plane $\mathrm{R}^{2}$, the value at point $P$ is the weighted average of the values at nearby 4 data point $P_{1}, P_{2}, P_{3}$ and $P_{4}$ (integer node). Denote the value of $F$ at $P_{i}$ by $F_{i}$ and $d_{i}$ be the distance between $P_{i}$ and the generic point $P$ in $\mathrm{R}^{2}$ (See Fig. 1). The result was established by the function:

$$
F(P)=\left[\sum_{i=1}^{4} F_{i} \prod_{j \neq i} d_{j}\right] /\left[\sum_{I=1}^{4} \prod_{j \neq i} d_{j}\right]
$$

3) Grand circle distance computation: the distance $d_{l}$ between two point on the earth $N_{l}$ and $N_{2}$ is given by the following formula: 


$$
d_{l}=R \cdot \arcsin \left\{\left\|\vec{P}_{1} \wedge \vec{P}_{2}\right\|\right\}
$$

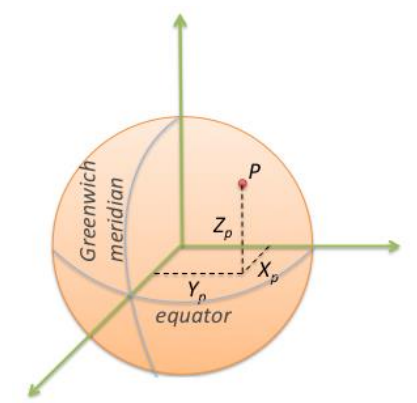

Fig.2. The Cartesian coordinates

Where $\vec{P}_{1}=\left(x_{1}, y_{1}, z_{1}\right)^{T} \vec{P}_{2}=\left(x_{2}, y_{2}, z_{2}\right)^{T}$ are the Cartesian coordinates of the nodes $N_{l}$ and $N_{2}, \wedge$ is the vector product and $R$ is the radius of the earth. For a given node $P$ (see Fig.2), the Cartesian coordinates are given by the following formula:

$$
\vec{P}=\left\{\begin{array}{l}
x=R \cdot \cos (\phi) \cdot \cos (\lambda) \\
y=R \cdot \cos (\phi) \cdot \sin (\lambda) \\
z=R \cdot \sin (\phi)
\end{array}\right.
$$

\subsection{Bellman Algorithm.}

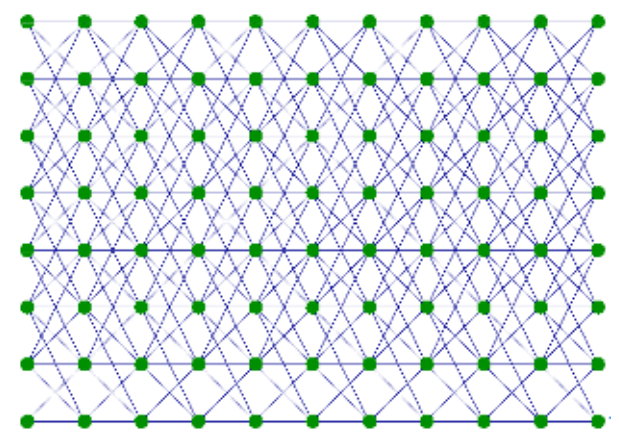

Fig 3. Graph used for the wind optimal trajectory design

In order to generate wind optimal trajectories, we start building a graph $G=\{\mathcal{N}, \mathcal{L}\}$ based on the wind grid (see Fig.3), for which the set $\mathcal{N}$ represents the nodes and $\mathcal{L}$ the links. Each node stores the following information: Latitude $\phi$, longitude $\lambda$, the east wind component $W_{E}$ and the north wind component $W_{N}$. ( see Fig.4)

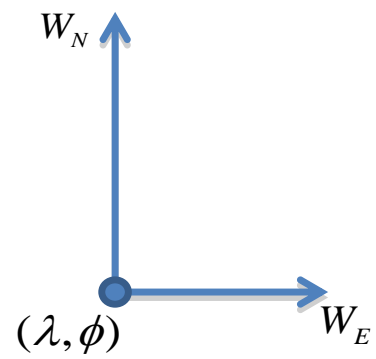

Fig 4. Information contained in each node

Each link contains also its associated bearing (see Fig.5) ${ }^{\theta_{l}}$, which is given by the following formula:

$$
\left\{\begin{array}{l}
\theta_{l}\left(N_{o}, N_{d}\right)=\arctan \left(\frac{y}{x}\right) \\
y=\sin (\Delta \lambda) \cdot \cos \left(\phi_{d}\right) \\
x=\cos \left(\phi_{0}\right) \cdot \sin \left(\phi_{d}\right)-\sin \left(\phi_{0}\right) \cdot \cos \left(\phi_{d}\right) \cdot \cos (\Delta \lambda) \\
\Delta \lambda=\lambda_{d}-\lambda_{o}
\end{array}\right.
$$




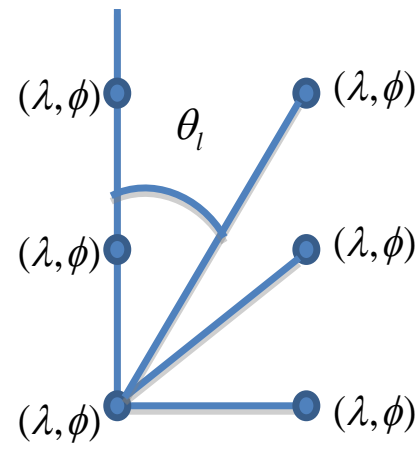

Fig 5. Information contained in each link

Tail wind $T W_{l}$ associated to each link is given by the following formula:

$$
\left\{\begin{array}{l}
T W_{l}=\frac{T W_{o}+T W_{d}}{2} \\
T W_{\mathrm{o}}=W_{\mathrm{o} N} \cdot \cos \left(\theta_{l}\right)+W_{\mathrm{oE}} \cdot \sin \left(\theta_{l}\right) \\
T W_{\mathrm{d}}=W_{\mathrm{d} N} \cdot \cos \left(\theta_{l}\right)+W_{\mathrm{d} E} \cdot \sin \left(\theta_{l}\right)
\end{array}\right.
$$

Where $T W_{o}$ and $T W_{d}$ are the tail winds related to original point and destination point.

To compute the wind optimal trajectory, we will consider for each link, the time needed by aircraft to connect node $N_{o}$ to node $N_{d}$. This time $t_{l}$ is given by formula:

$$
t_{l}=\frac{d_{l}}{T_{a}+T W_{l}}
$$

Once applying Bellman algorithm on this specially structured gird, the minimal time cost path between any points at very right $\left(N_{o}\right)$ and all the points at very left $\left(N_{d}\right)$ can be found. The algorithm is organized into three steps:

STEP 1: Initialization This step initializes time cost (cost) from source to all vertices as infinite and distance to source itself as 0 .

STEP 2: Propagation The source node is first considered and its associated neighboring links.

Starting from the source node $N_{o}$ (first column) and for each link associated to $N_{o}$ the algorithm marks the neighboring nodes of $N_{o}$ with the following rule:

- if $\operatorname{cost}\left[N_{d}\right]>\operatorname{cost}\left[N_{o}\right]+t_{l}$ then update ${ }^{\operatorname{cost}\left[N_{d}\right]}$ to ${ }^{\operatorname{cost}\left[N_{d}\right]=\operatorname{cost}\left[N_{o}\right]+t_{l}}$ (keep in node $N_{d}$ the node $N_{o}$ which has been use for this update)

Shift to the next column (column 2) and apply the same rule to all nodes, which have been updated in order to propagate the distance update to the third column. This process is repeated until the propagation reaches the last column (on the right).

STEP 3: Path building If one want to compute the shortest path for the source node $N_{o}$ to any destination nodes on the right $N_{d}$, one first select a destination node among the nodes belonging to the last column (column number $K$ ). Select the node $N_{K-1}$ in column $K-1$ that has updated the $N_{d}$ node in column $K$. Then, select the node $N_{K-2}$ in column $K$ - 2 which has updated the node $N_{K-1}$ in column $K$ 1 and so on until the source node is reached in this back propagation process.

For each weather sample, such minimum time path algorithm is computed in order to create a set of wind optimal trajectories that has to be clustered.

\section{Wind Uncertainty Quantification}

\subsection{Gray Mathematics Theory}

The Gray systematic theory regards random variables as Gray quantities which change constantly in certain scope, and also regards stochastic process as a time related Gray process which changes constantly in certain scope [7].

Suppose a group of measurement data to list as $X=\{x(k), k=1,2, \ldots, n\}$, the uncertainty quantification process is divided into three steps: 
1) Arrange this sequence in turn from small to big, we obtain $X^{(0)}=\left\{x^{(0)}(k), k=1,2, \ldots, n\right\}$ where $x^{(0)}(k) \leq x^{(0)}(k+1), k=1,2, \ldots, n-1$

2) Accumulate the elements of sequence $X^{(0)}$ in turn and a new sequence $X^{(1)}$ originates, the form of which is as follows:

$$
\begin{aligned}
& X^{(1)}=\left\{x^{(1)}(k), k=1,2, \ldots, n\right\}=\left\{x^{(1)}(1), x^{(1)}(2), \ldots, x^{(1)}(\mathrm{n})\right\} \\
= & \left\{x^{(1)}(1), x^{(1)}(1)+x^{(1)}(2), \ldots, x^{(1)}(1)+x^{(1)}(2)+\ldots+x^{(1)}(\mathrm{n})\right\}
\end{aligned}
$$

3) Uncertainty quantification:

$$
\left\{\begin{array}{l}
\Delta(k)=\frac{x^{(1)}(n)}{n} k-x^{(1)}(k) \\
\Delta \max =\max (\Delta(k), \Delta(k), \ldots, \Delta(k)) \\
s=c \frac{\Delta \max }{n} \\
\bar{x}=\frac{1}{n} \sum_{1}^{n} x^{(0)}(i)
\end{array}\right.
$$

$c$ is the Gray constant coefficient ${ }^{10}$, which is generally taken as $2.5 ; \mathrm{s}$ is a quantitative evaluation to uncertainty of data based on the Gray evaluation method.

Then the interval $[\bar{x}-s, \bar{x}+s]$ could be taken as the estimated interval of the true value.

\subsection{Wind Data Interval Quantification}

Each wind grid point has 34 groups of wind date. By applying uncertainty quantification method, the interval of wind speed on each grid point is determined. (See Fig.6) Taking 41 points on altitude -40 degree as an example, the black line is average of all the wind speed, the blue lines are the original data boundaries, the red lines are the interval quantified by grey mathematical theory. Note that the interval is quite concentrated since the original data is not very dispersed.

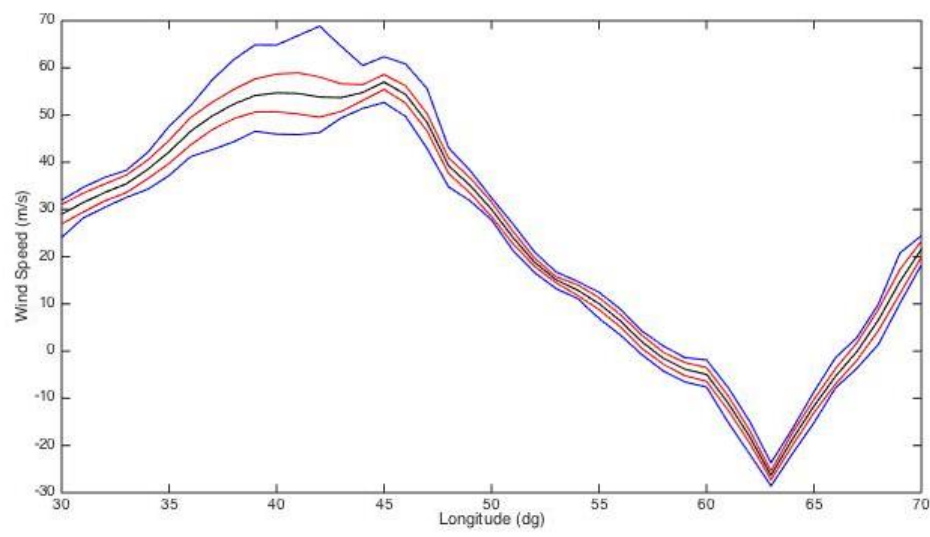

Fig 6. Determination of the estimated interval for wind data based on Gray mathematical theory

\section{Optimal Trajectory Interval Computation}

The optimal trajectory interval is found, by combining Bellman algorithm with wind speed interval. The wind gird $G=\{\mathcal{N}, \mathcal{L}\}$ is similar with the certain optimization process.

The information contains in each point $N$ : latitude $\phi$, longitude $\lambda$, east wind interval $\left[W_{E_{\min }}, W_{E_{\max }}\right]$ and north wind interval $\left[W_{N_{\min }}, W_{N_{\max }}\right]$.

The information contains in each link $l \in \mathcal{L}=\left(N_{o}, N_{d}\right)$ : Original point $N_{o}$, destination point $N_{d}$, distance between two point $d_{l}$, the link associated bearing $\theta_{l}$, tail wind interval related to link $\left[T W_{l_{\min }}, T W_{l_{\max }}\right]$ and link time consumption interval $\left[t_{l_{\min }}, t_{l_{\max }}\right]$. 


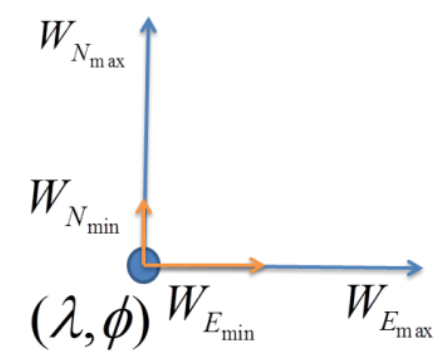

Fig 7. Information contained in each node

$t_{l_{\min }}$ and $t_{l_{\max }}$ are given in the following formula:

$\left\{\begin{array}{l}t_{l_{\min }}=\frac{d_{l}}{T_{a}+T W_{l_{\min }}} \\ t_{l_{\max }}=\frac{d_{l}}{T_{a}+T W_{l_{\max }}}\end{array}\right.$

Based on all the time consumption $t_{l_{\min }}$ and $t_{l_{\max }}$, by applying Bellman algorithm, two optimal paths are generated. The optimal trajectory interval can be determined by those two paths. The overall processing can be summarized by the figure 8 .

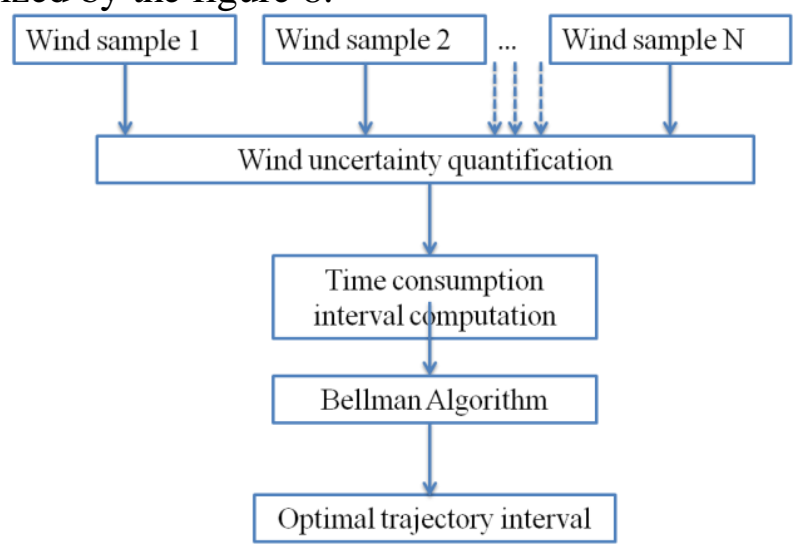

Fig 8. Overall structure of the algorithm

\section{Result}

This section presents the initial results that have been produced by this new algorithm. First we have considered two wind samples over the Atlantic Ocean from January 09, 2016. An example of such map is given on figure 9.

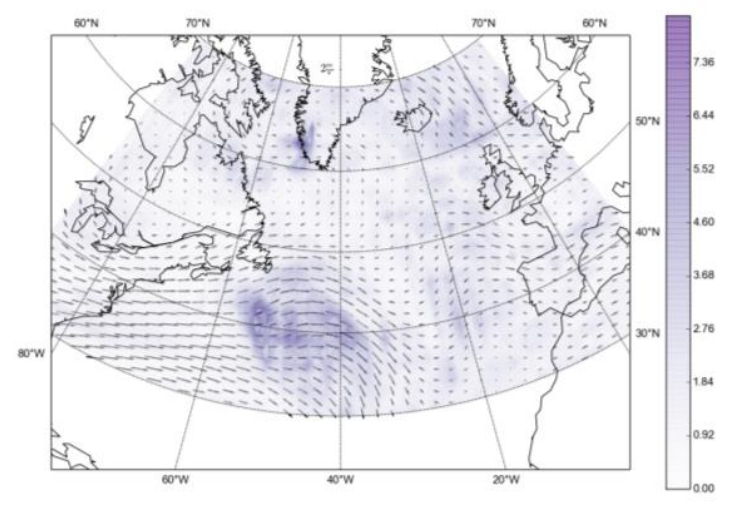

Fig 9. Example of wind distribution over the Atlantic Ocean

Aircraft cruise speed is 400 knots, flight starting point is $\vec{P}_{\mathrm{o}}$ (latitude=30, longitude=-90), final point is $\vec{P}_{\mathrm{d}}$ (latitude=60, longitude=10). The figure 10 and figure 11 give the trajectories computed by 
certainty method and interval method. In the pictures, the blue paths are certainty trajectories and the red paths are the trajectory interval.

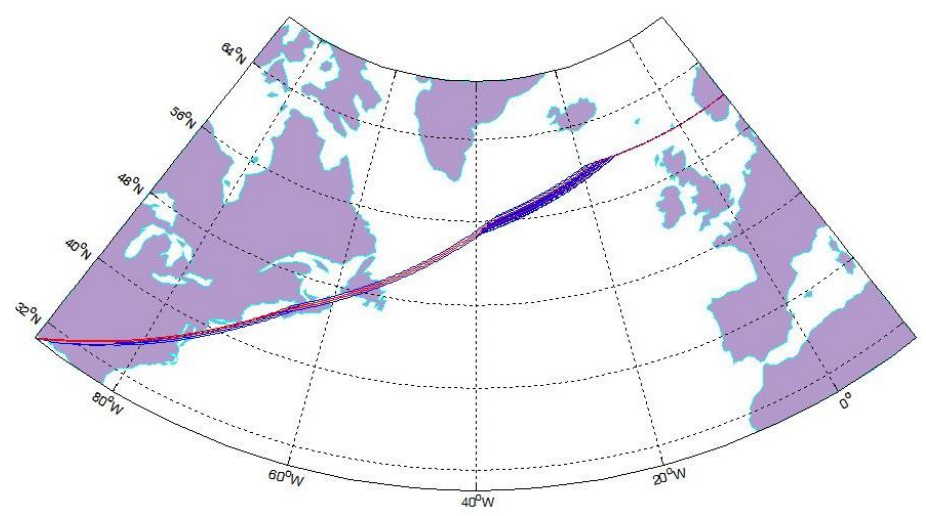

Fig 10. 34 optimal trajectories and the interval of optimal trajectories

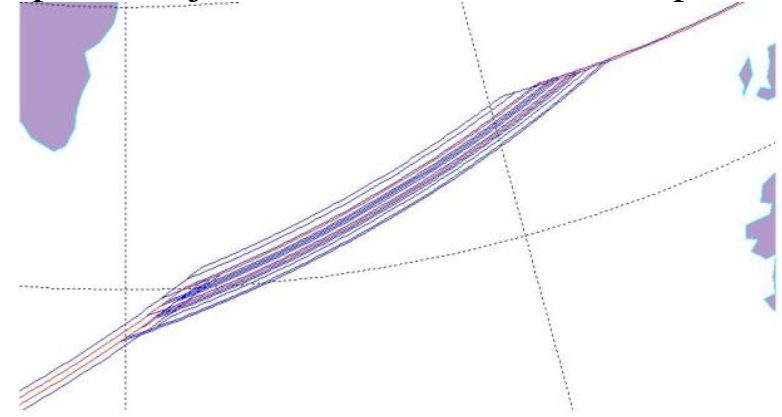

Fig 11. 34 optimal trajectories and the interval of optimal trajectories (partial magnified)

In figure 12 and figure 13, the black path is the trajectory without consideration of wind effect, the red paths are the trajectory interval, and the blue path is optimal trajectory generated by using average wind speed.

Regarding the time consumption, trajectory interval has minimal time 32506s and maximal time 33493s, which cover all 34 certainty trajectories' time consumption.

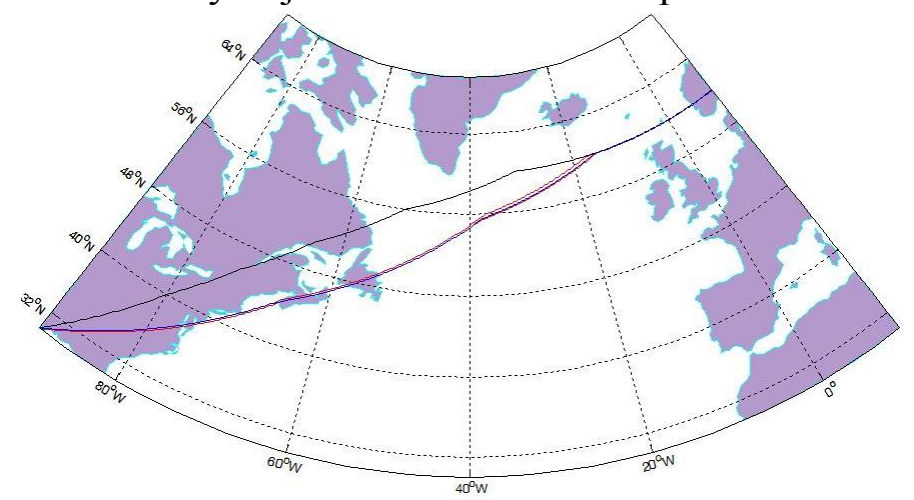

Fig 13. 4 different particular trajectories

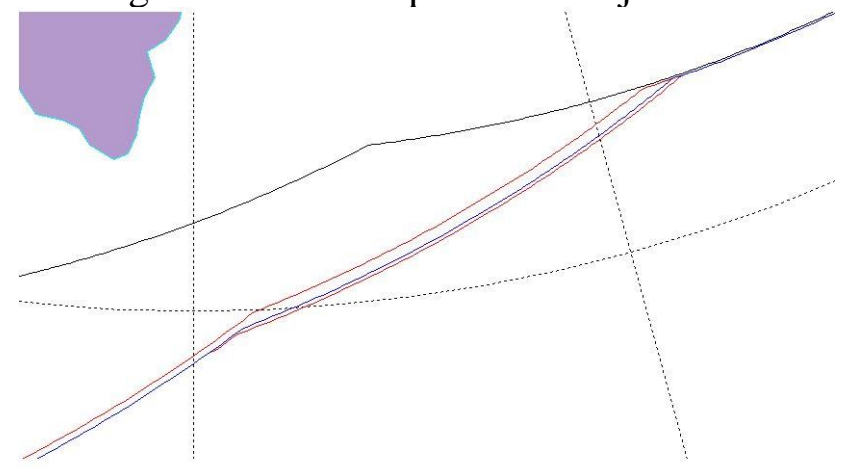

Fig 14. 4 different particular trajectories (partial magnified) 


\section{Conclusion}

This paper has introduced a new approach for designing wind optimal trajectory. First of all, a grid network contains wind data has been introduced. Wind has been interpolated on a more accurate grid, and then tail wind on each link has been computed. Secondly, the wind speed interval on each point has been determined by applying gray mathematics theory. Based on the classical bellman algorithm, the optimal trajectory interval has been generated. This new methodology has been successfully applied to real wind data in order to identify wind optimal trajectory interval.

\section{References}

[1]. Dijkstra E. A note on two problems in connexion with graph. Numerische Mathematlk, 1:269-271, 1959

[2]. Hart P, Nilsson N, and Raphae B. A formal basis for the heuristic determination of minimum cost paths. IEEE Transactions on Systems Science and Cybernetics, 4(2):100-107, 1968

[3]. Farin G. Level Set Methods and Fast Marching Methods: evolving interfaces in computational geometry, fluid mechanics, and computer vision, and materials science, volume 3. Cambridge University Press, 1999

[4]. Pe^tre`s C, Pailhas Y, Patron P, Petillot Y, Evans J, and D. Lane. Planning for autonomous underwater vehicles. IEEE Transactions on Robotics, 23(2):331-341, 2007

[5]. Alton K. Dijkstra-like Ordered Upwind Methods for Solving Static Hamilton-Jacobi Equations. $\mathrm{PhD}$ thesis, The University of British Columbia, 2010

[6]. K. Legrand, S. Puechmorel, D. Delahaye and Y. Zhu. Aircraft Trajectory Planning under Wind Uncertainties. In Digital Avionics Systems Conference (DASC), 2016

[7]. Xiaojun. W and Lei. W. Uncertainty quantification and propagation analysis of structures based on measurement data. Mathematical and Computer Modelling, 54 (2011) 2725-2735

[8]. Moore R. E. Methods and applications of interval analysis [M]. London: Prentice-Hall, Inc., 1979

[9]. Shepard D. A two-dimensional interpolation function for irregularly- spaced data. In ACM Conf. ACM, 1968

[10]. H.B.Zhang, Z.Y.Wang, Z.M.Liu, on the verification of measurement uncertainty. Acta Metrologica Sinica 28(3) (2007)193-197. 